\title{
Performance of a Digital Video Camcorder for the Autonomous Biological System Experiment onboard Space Station Mir
}

\author{
Masamichi Yamashita ${ }^{1}$, Masaaki Sasada ${ }^{2}$, Katsura Sugiura ${ }^{3}$, Yoji Ishikawa ${ }^{4}$, Kensei Kobayashi $^{5}$, \\ Hiroshi Mizutani $^{6}$, Yukishige Kawasaki ${ }^{7}$, Junpei Koike ${ }^{8}$, Kenichi Ijiri ${ }^{9}$, Jane Poynter ${ }^{10}$, Taber MacCallum ${ }^{10}$ \\ and Grant Anderson ${ }^{10}$ \\ ${ }^{1}$ Institute of Space and Astronautical Science, Sagamihara, 229-8510, Japan \\ 2 Tokyo Broadcasting System, Akasaka, Minato-ku, Tokyo, 107, Japan \\ 3 Sagami Woman's University, Sagamihara, Japan \\ ${ }^{4}$ Obayashi Corporation, Kiyose, Tokyo, 204-0011, Japan \\ 5 Yokohama National University, Hodogaya-ku, Yokohama, 240, Japan \\ 6 Nihon University, Kameino, Fujisawa, 252-8510, Japan \\ ${ }^{7}$ Mitsubishi Kasei Institute of Life Science, Minami-Ohya, Machida, 194, Japan \\ 8 Tokyo Institute of Technology, Nagatsuta, Yokohama, 226, Japan \\ ${ }^{9}$ University of Tokyo, Yayoi, Bunkyo-ku, Tokyo, 113-0032, Japan \\ ${ }^{10}$ Paragon Space Development, Tucson, Arizona, USA
}

\begin{abstract}
A video imaging and recording system was utilized in the Autonomous Biological System experiment onboard the space station Mir. Video image of the mini-ecological system was successfully recorded. The whole system was retrieved to the ground after its operation in orbit for four months. Performance of the video system is summarized here together with technical problems encountered. Defects of pixel had been developed in the imaging device. Cause of these defects could be attributed to its exposure against space radiation. Auto white balance was another function of the camcorder that was deviated from normal range of its performance once in orbit but recovered to normal after a while. Possible use of imaging devices for dosimetry is proposed to record space radiation environment at the site close to the experiment.

Key words; CCD, semiconductor, space radiation, video system
\end{abstract}

\section{Introduction}

Imaging is a powerful method of science in many disciplines, and accepted as a generic tool for space experiment. A great amount of efforts has been paid to develop sensors for the mission of space astronomy and earth observation that takes under the harsh environment of outer space, including cosmic radiation. In parallel to this kind of technology dedicated to space uses, highly advanced imaging devices are available for ground use, and could be implemented on space crafts. Quality of image and system functions have been so well equipped to the imaging system at a level of the ordinary consumer products. If those products could be utilized in space experiment, they could give far more advantages against the mission-dedicated system. One of the technical issues that should be solved for implementation of consumer products is that we have no confident experience of sending such video system to space longer than weeks. For their use in the coming space station era, it is necessary to

Original Paper

Received: November 20, 1998.

Accepted: December 15, 1998.

Address for correspondence: Masamichi Yamashita yamashita@surc.isas.ac.jp evaluate their performance and durability under the space environment. Here is a report on operation of a video camcorder operated in space for a period of four months.

\section{Video Filming of Autonomous Biological System: System and Operation}

A video camcorder was operated onboard the space station Mir to record the Autonomous Biological System experiment (MacCallum et al., 1998.) It took video image of the ecological system consisting of plants, animals and other living organisms grown and transformed in the sealed container (Ijiri et al., 1998). The video image was recorded on a magnetic tape during the mission.

The camcorder implemented in this experiment was a model commercially available at a category of consumer products. It was a mini Digital Video, miniDV, camcorder DCR-PC7 (Sony, Japan.) Several minor portions were modified of this camcorder. As shown in Fig. 1, appendage items, such as a cover on connectors, a liquid crystal monitor, and a rubber piece on the viewfinder, were removed from its body. In order to start and stop recording automatically, the control software stored in an electronically programmable read only memory chip was replaced by a modified program. This modification of the software enabled to select several default setting of the unit different from those for the commercial model. The default 
setting, optimized for the experiment, was loaded to the camcorder when power was supplied to it. The miniDV tape loaded on the camcorder was essentially the same as a commercially available one, except a minute longer in its length than that of nominal 60 minutes.

Video-filming was activated at least once a day all through the mission, either on Space Shuttle after the launch till its docking to Mir, onboard the space station Mir, or another Space Shuttle for retrieval to the ground. The duration of recording at a time was selected to either two minutes or several seconds. The timeline of recording was composed on the basis of scientific priority defined and operational considerations including the risk management. Total length of recording in orbit was designed to fill a full length of the tape, i.e. 64 minutes. Time slot of filming was placed during the light phase given to the Autonomous Biological System. Filming under dark was not feasible by a technical reason in the control logic developed for the experiment. The light source, a fluorescent lamp, was located behind the two sample cylinders. A chart of standard color tiles was placed in the midst of the two cylinders, in order to calibrate the color of recorded image at the post flight analysis.

All the time sequences of filming were registered in the memory of the control unit and executed at its time mark. In case of the electric power outage that would happen at a planned time for recording, an alternate filming session was initiated as soon as power was recovered. Since the control of the Autonomous Biological System could be overridden by a manual commanding made by a crew, he/she could initiate additional picturing session in orbit. The ground control unit of the experiment system run with the same procedure and hardware in parallel to the flight experiment.

From the inspection of the recovered tape, performance of the video filming system in orbit was satisfactory. It was confirmed to fill the scientific requirements defined. The filming sequence was executed as planned, with one minor exemption caused by a cutoff of electric power supply from Mir one day in the middle of the mission. One additional picturing session was activated by a crew after it was transferred to Space Shuttle for the retrieval. Those off-nominal sequences were executed correctly. Image quality was sufficient to make scientific analysis on the recorded images. The automatic adjustment of iris opening and the electronic amplification of video signal amplitude worked fine to take picture at different light intensity of the image.

Even function of the retrieved camcorder was in the range of normal, two technical issues were found in the video image taken in orbit and the camcorder.

One was a defect of pixels that were found in the retrieved camcorder. It is so-called "white spot" that is caused by an increase in dark level of the pixel. This defect was originated by the exposure of the imaging device to space environment, and it was irreversibly damaged.

The other issue was the white balance function of the camcorder. It was disturbed during a period in orbit, but recovered afterwards. In the following sections, those two technical items are described in detail.

\section{Technical Evaluations on Video Camcorder}

\section{Distribution of Dark Level and Defects of Pixel at Exposure to Space Environment}

\subsection{Background}

Radiation environment in space is known to cause damages on electronics element even at low earth orbit. Electronic system and components, as same as living organisms, are protected on ground by the thick layer of atmosphere and the magnetic field of earth. For application of electronics products on the ground, there exists no requirement for durability against radiation except in the special case such as in nuclear plants. In order to employ ordinary ground products in space experiment, feasibility of usage under space radiation should be well examined for those.

Chips of Charge Coupled Device, CCD, are commonly adopted in electronic imaging systems. It has a huge number of imaging pixel that is uniformly structured and processed. Electronic properties of each unit can be expressed with the analog level of dark, the sensitivity to light intensity, and thermal dependence of those properties. Among many kinds of electronics element, CCD is a good subject to evaluate the radiation effects on semiconductors and the elementary processes that cause the defects. In recent years, extensive studies have been conducted on CCD chips both in space and on ground (Sasada et al., 1998.) Technology development is quite active in the silicon process of CCD chips. As a trend of engineering challenge, number of pixels has been increased at reduction of its physical size in each pixel. The smaller the pixel's size, the larger the radiation damage is resulted.

Imaging system will be somehow a generic hardware for many scientific researches in the space station era. It is important to evaluate tolerance of imaging device under space environment, and examine the elementary process

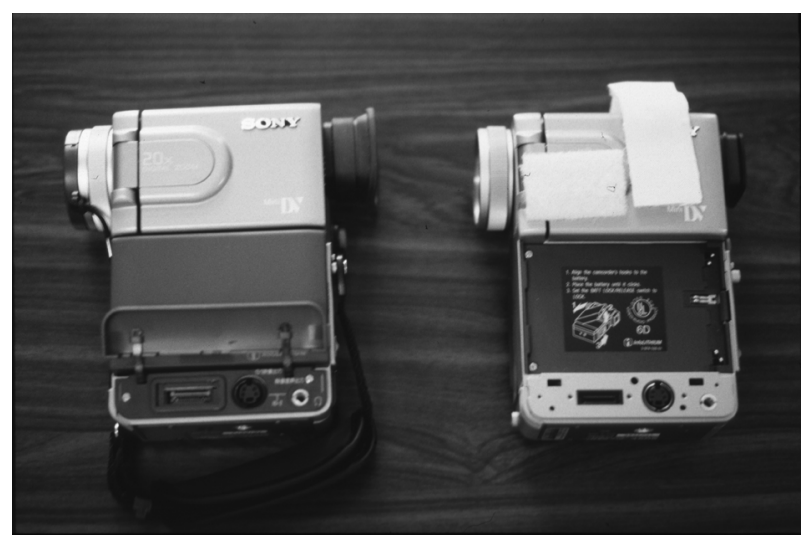

Fig.1 Digital Video Camcorder implemented for the Autonomous Biological System experiment onboard Space Station Mir Minor modification was made on the fllight camcorder (right) which is available as a consumer product (left.) 
relevant to the radiation damages on electronics chips. For their application on space stations, study should be focused on the characteristics of chips and system exposed to space radiation environment for a period of years. We might require finding and defining fundamental features of damages of CCD chips operated on spacecraft.

One of outcomes from the present evaluation is to determine the requirements of logistics for keeping the video system functional in orbit, and to define the requirement to replace imaging device at a certain interval. Once we could know which component of space radiation makes such damage of CCD and causes propagation of damages, the imaging devices could be utilized as an alternate dosimeter. By taking black image with iris closed periodically in orbit, it might provide time history of radiation dose through the period. If imaging device would be equipped close to biological specimen, it could be utilized as a dosimeter for those specimens. In order to do so, camcorder should be capable to close iris by a remote command at taking radiation data.

\subsection{Method}

Dark level of video signal at each pixel was measured with the lens closed, in order to shut light onto the CCD chip. Two units of camcorders, Sony DCR-PC7, were subjected to this evaluation of dark level. One was implemented on Autonomous Biological System flight experiment, and retrieved after the four months operation on Mir. The other was the ground control camcorder, which had been stayed on the ground.

Measurement of the dark level and data processing were executed by Video Measurement Set (Tektronix, VM 700A). The signal analyzed was Y signal from S-video output of the camcorder. Video signal was averaged over 32 frames as a preconditioning for the data reduction. Ambient temperature was at $25^{\circ} \mathrm{C}$. Since the dark level of CCD pixel is quite sensitive to the thermal properties of the semiconductor associated, temperature of the outer casing of the camcorder was measured and logged during

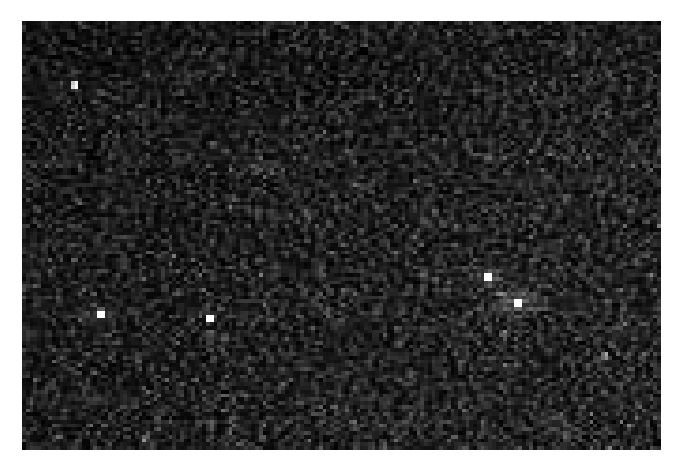

Fig.2 Amplified black image of the flight camcorder. Note that several white, red, and blue points seen in this image are defects of CCD pixel caused by its exposure to space environment for four months. Amplification of the image is roughly four times. Even each single frame looks noisy as shown here, the integration of several frames by the neural system of human makes it plain black. the test. The electronic gain of CCD signal was confirmed to be automatically increased to $+18 \mathrm{~dB}$ at taking image with the lens closed.

The picture taken and recorded on the miniDV tape by the camcorders was transferred to a personal computer. To convert an image file in a format of film strip, a personal computer, Power Macintosh G3 MT (Apple) with a DV capture card, FireMax-2 (ProMax), was configured to transfer the digital video data directly through the IEEE 1394 interface. The image file acquired was processed and analyzed quantitatively by software, Premiere and Photoshop (Adobe.)

\subsection{Results}

\section{General Performance}

Quality of the images taken and recorded by the flight camcorder was satisfactory for the scientific purpose of observation on Autonomous Biological System. Patterned dark stripe either in horizontal or vertical axis, and dark spot area at left end zone are seen in the captured pictures when the lens of the camera unit was closed. However, these patterns were not distinguishing from the normal range, and found to be acceptable for the analysis. Distinctively damaged pixels were surveyed among the video image taken in orbit. It was hard to find such defects in the recorded image, even location of the defects are known by the picture of the plain black image. The process of image compression might sweep such dot-sized image of damaged pixel away, in case the image has rather complex features.

The function of taking picture, recording on tape and any other features activated at the post flight test were found to be normal, and none was deviated from the stated function of the camcorder. The characteristics of video signal, such as the voltage level of Sync pulse was found to meet the specification of NTSC video signal. Chroma sync waveform was not in its specified range. Even it resulted in the failure of auto synchronization of chroma

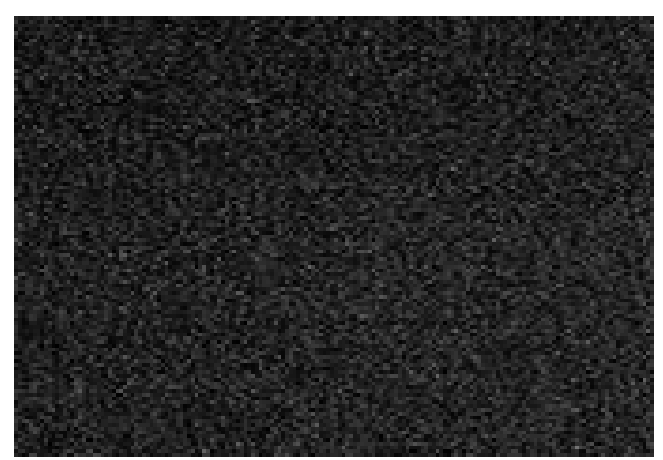

Fig.3 Amplified black image of the ground camcorder. Amplification is roughly four times in intensity of the original color image. Patterned dark stripe and spot in the frame are seen. Darker stripe at left end of the image is originated by the temperature gradient formed in the CCD chip. Left end of CCD seems to be the coldest part in the chip. 
part of signal by the system used at the test, it did not affect the results of this evaluation on dark level that dealt only Y part of the signal.

\section{Damaged Pixels}

The ground control unit gave a plain black image when a cap closed its lens. Dark image from the flight camcorder showed many dots at single pixel size with color of white, red or blue. There were found no adjacent two pixels that showed such defect. In order to display how dark level of pixel distributes, the image signal is amplified four times in Figs. 2 and 3, for the flight and the ground camcorder, respectively.

The distribution of dark level was analyzed after image data were digitized and averaged over a definite number of frames. The ground camcorder had 5 pixels that exceed 3 IRE. The brightest pixel was at a level of 2.6 IRE. Distribution of the IRE number among the pixels took a normal statistical feature. IRE is a unit that was defined by Institute of Radio Engineers, the antecedents of IEEE. Zero IRE and 100 IRE correspond to black and white at each extreme, respectively.

The flight camcorder showed 363 pixels that exceeded 3 IRE just after power was turned on. The biggest number, i.e. the brightest pixel, was 40.8 IRE. IRE number for the flight camcorder was dispersed widely for damaged pixels, being overlapped to a narrow peak distribution for normal pixels.

Effects of Data Compression for Image of Damaged Pixels

Image data taken by CCD is compressed before it is recorded on a miniDV tape. It was evaluated how much image data is degraded by this compression. Number of damaged pixel in the processed image was compared to that in the original image came out directly from the camcorder without compression or recording. Number of pixel that exceeded 8 IRE was found 487 in the real time video output. This number was reduced to 415 in the video image replayed from the recorded tape. Dot image was kept the same in the replayed frame. The compressed image at this test was essentially plain black. MiniDV image compression might give less degradation for the image of fine dots embedded in a plain black background.

\section{Temperature Dependence of Black Level}

Temperature at the outer casing of the flight camcorder reached to an equilibrated level after 90 minutes from the power-on. The equilibrated temperature on the surface was $10.2^{\circ} \mathrm{C}$ higher than ambient. Number of pixels higher than 16 IRE was 43 at the beginning, and increased to 206 after 150 minutes from the start. The brightest number of IRE also increased from 40.8 IRE to a level more than 100 IRE, i.e. the saturated value.

In ordinary camera units, temperature dependence of black level is compensated by the signal from a part of CCD chip where pixel is masked against light. Level of signal from that part has the same temperature dependence with other active pixels. Because of this capability to compensate thermal effects, black level of the processed video signal is not affected by temperature much. However, signal level of the damaged pixels shifted brighter when the CCD chip was warmed up.

As a subsidiary effect of the thermal properties, following tendency was found in the image taken in this test. Signal level was 8 to $9 \%$ lower at the left end of the frame when the camcorder was warmed up by its internal power dissipation. The dark level was lower by a similar degree along the vertical axis of the image at the left end. It is suspected that right hand side of the CCD chip is located either to a cold part in the camcorder unit, or is close to a heat conducting component around the chip.

\subsection{Conclusion}

Severe damages in the CCD chip were found in the flight camcorder. The damaged pixels were visibly presented in the image that was taken with the lens closed. These damages are related to the exposure of CCD chip to space radiation environment in low earth orbit for a period of four months. In contrast to the flight camcorder, no defect was found on the image taken by the ground control camcorder. The dark level of pixel output is highly sensitive to temperature. It should be noted that even at a Space Shuttle mission of one week, there might be about 20 pixels damaged exceeding a threshold level of 3 IRE. This could be a quite distinguishable number compared to 5 found in the ground control camcorder. If the experiment could be repeated in orbit to examine the time course of the generation and the propagation of damages, we can know whether it would be linearly accumulated with the dosage of space radiation or not. Effective type of radiation to cause defects or damage on CCD pixel has been extensively studied with simulated exposure experiment on the ground with several kinds of radiation source, such as heavy highenergy particles and neutrons (Kotov et al. 1998.) By comparing camera units, which are shielded in different ways to take image, we can assess which component of space radiation is the most critical to the damage in imaging device.

Number of defect pixels increases when camcorder is warmed up. Evaluation of a damaged pixel is feasible with replayed video signal that is compressed before its recording on miniDV tape. This makes in-orbitexamination feasible to study propagation of damages without real time data collection. At the same time, these features, together with knowledge on sensitivity of the element against space radiation, opens the way to utilize CCD camcorder for a convenient radiation monitor system for space biology experiment.

\section{Auto White Balance}

\subsection{Background}

White balance is a function of imaging systems to process color data by adjusting image of white object to white at its appearance. Camcorder, in the category of consumer products, provides several modes of white balance, such as fixed to a typical color temperature of light, manual set by taking an image of white subject, and 
automatic adjustment of white balance. In the Autonomous Biological System experiment, the mode of white balance was set to "auto" mode. Because this function of auto white balance is achieved by the software implemented on the camcorder, we can only make a guess on its performance, and how it is done. The camcorder, Sony PC7, seems to have an algorithm as follows. When camera power is turned on with a set of 'auto exposure', opening of its iris is adjusted to make the brightest part in the image be at the maximum level of the video signal. Then the most bright and wide area in the image is turned to white by modifying the color components of the whole video signal. The factor for color adjustment is limited, in general, to cover a range of color temperature from $2500 \mathrm{~K}$, for a tungsten lamp, to $6000 \mathrm{~K}$, of the sunlight. The camcorder continues this color adjustment, while the camera is kept on and set to the auto white balance. By this continuous correction of color balance, white object could be kept as white in the image frame when it is brought from indoor to outdoor scene, or vice versa.

In the Autonomous Biological System experiment, color of the image turned to greenish at a shot taken on 3 January 1998. At this occasion, the iris was fully opened at the decrease in light intensity of the image. The electronic gain of video image was increased to $+3 \mathrm{~dB}$ at the beginning, and returned to $0 \mathrm{~dB}$ shortly after the start. From the time mark recorded on the shot, and the log of power supply from Mir, it was identified as the shot taken after a power outage that lasted for hours on the day. Since this power outage was associated with a malfunction of the whole Mir system, several sources of disturbance could be speculated to explain the change in white balance. Greenish color had stayed in the following days for a while, and returned normal once. It turned greenish again on 13 January.

\subsection{Evaluation}

In order to evaluate the normal range of shift in color data for adjusting white balance, values of red, green and blue components of the video signal were determined quantitatively. Photoshop analyzed the image data at the standard color chart placed in the middle of the viewing field. Two successive frames taken on 2nd and 3rd January 1998, the image got greenish at that time, were subjected to this analysis.

In a part of the image where reflected light from lamp dominate, red, green and blue values were found all 255 as being saturated. The change of color component was, in terms of R, G, and B numbers at the standard color chart, $20 \%$ increase in green, and $20 \%$ decrease in red.

The function of auto white balance in the camcorder was tested to take image of a white paper and a plain paper colored to $20 \%$ greenish. Both images were appeared to be white when they were processed by auto white balance. The camcorder was found to be capable to adjust white at such a degree of deviation in color. It can be concluded that greenish color recorded in orbit was not in the normal range of the auto white balance capability of Sony DCRPC7 camcorder.

\subsection{Conclusion}

Function of auto white balance is fully controlled by the software developed for the camcorder. It was found that the retrieved flight camcorder functioned normally including the feature of auto white balance. Temporal malfunction in the control circuit could happen at latch up of memory element, or other part in integrated circuits at a hit of cosmic ray. However, greenish color did not recovered by the power-off reset procedure of the camcorder. There could be a memorized white balance parameter that was affected by irradiation temporarily. Without further technical information disclosed by its manufacturer, our evaluation on this matter would be a limited one as discussed here. We should also consider a possible change of color temperature of fluorescent lamp at that time in orbit.

\section{Concluding Remarks}

Increase of dark level was found at pixel level in the CCD of the flight camcorder. There was a thermal effect on the dark level at the damaged pixels. The number of visible spots of damage increased when camcorder was warmed up. Those damage could be examined from the image taken with the iris or the lens fully closed, and recorded on miniDV tape. It shows feasibility of video camcorders to utilize it for convenient dosimetry in space experiment. It provides time history of exposure against space radiation by taking black images periodically in orbit. It was found a minor problem of white balance in the recorded image happened onboard Mir. It requires further investigation in order to clarify the source of deviation.

\section{Acknowledgments}

Modification of the Digital Video camcorder was made by a courtesy of Sony USA.

\section{References}

Ijiri, K., Mizuno, R., Narita, T.,Ohmura, T., Ishikawa, Y., Yamashita, M., Anderson, G., Poynter, J. and MacCallum, T. (1998) Behavior and Reproduction of Invertebrate Animals during and after A Long-Term Microgravity: Space Experiments Using An Autonomous Biological System (ABS) Biol. Sci. Space, 12, 377-388.

Kotov, Y.D., Zhamoycin, D.M. , Popov, V.D. and Sasada, M., Dark Currents in CCD Induced by Single Interactions of 14.9 MeV Neutrons, IEEE Trans. Nucl. Sci., in print (Dec, 1998).

MacCallum, T., Poynter, J. and Anderson, G. (1998) Autonomous Biological System Experiment, in this issue, Biol. Sci. Space, 12, 363-365.

Sasada, M., Saito, T., Fujii, M. and Zelentchikov, N.I. (1995) Damaging of the CCD Cameras onboard the Mir Manned Space Station, Proc. Space Utiliz. Symp., 12, 123-126. 